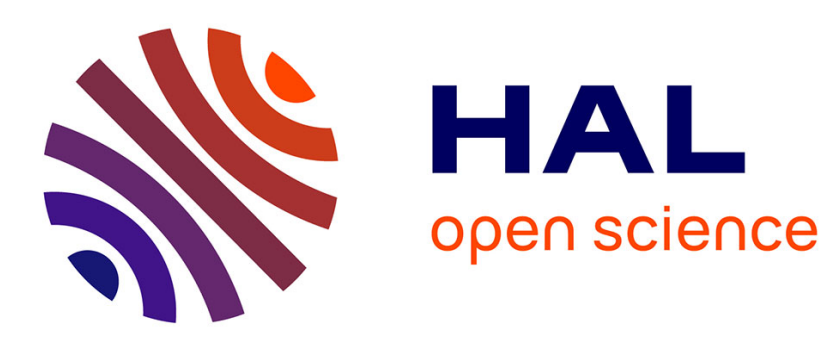

\title{
Soft Actuation of Structured Cylinders through Auxetic Behavior
}

\author{
Arnaud Lazarus, Pedro M. Reis
}

\section{To cite this version:}

Arnaud Lazarus, Pedro M. Reis. Soft Actuation of Structured Cylinders through Auxetic Behavior. Advanced Engineering Materials, 2015, 17 (6), pp.815-820. 10.1002/adem.201400433 . hal-01447311

\section{HAL Id: hal-01447311 https://hal.science/hal-01447311}

Submitted on 25 Nov 2017

HAL is a multi-disciplinary open access archive for the deposit and dissemination of scientific research documents, whether they are published or not. The documents may come from teaching and research institutions in France or abroad, or from public or private research centers.
L'archive ouverte pluridisciplinaire $\mathbf{H A L}$, est destinée au dépôt et à la diffusion de documents scientifiques de niveau recherche, publiés ou non, émanant des établissements d'enseignement et de recherche français ou étrangers, des laboratoires publics ou privés. 


\title{
Soft Actuation of Structured Cylinders through Auxetic Behavior**
}

\author{
By Arnaud Lazarus and Pedro M. Reis*
}

There has been a recent upsurge in the study of auxetic, or negative Poisson's ratio, materials that derive functionality from mechanical instabilities of the underlying structure. ${ }^{[1-5]}$ One of the simplest auxetic designs comprises an elastomeric slab patterned with an array of voids. ${ }^{[6,7]}$ Under uniaxial compression, the slender ligaments contiguous to the voids buckle cooperatively, resulting in the shrinkage in the orthogonal direction, and hence a negative Poisson's ratio. ${ }^{\left[{ }^{8-11]}\right.}$ This mechanism has also been implemented in patterned spherical shells that can significantly reduce their volume under loading, while remaining spherical. ${ }^{[12]}$ We regard this self-folding induced by buckling as a minimal mechanism for soft actuation, with a single radial degree of freedom.

Soft actuators, as opposed to those with rigid parts, have been gaining prominence in robotics. ${ }^{[13,14]}$ Their ability for distributed deformation with many degrees of freedom enhances flexibility, dexterity, and environmental adaptivity. ${ }^{[15,16]}$ A variety of mechanisms have been explored for soft actuation, ${ }^{[17]}$ including: electro-active polymers, ${ }^{[18,19]}$ electroor magneto-active fluids, ${ }^{[20-22]}$ shape-memory alloys, ${ }^{[23,24]}$ inflatable architectures, ${ }^{[25-28]}$ and granular jamming. ${ }^{[29,30]}$ Still, realizing soft structures with many degrees of freedom, such as in the arms of an octopus, ${ }^{[16,18,31]}$ remains challenging given the large number of embedded activators required. ${ }^{[29]}$

Here, we introduce a novel design for soft mechanical structures that exploit the auxetic behavior of cylindrical shells that are patterned with an array of voids, to achieve reversible flexural and twisting motion, as shown in Figure 1a

[*] P. M. Reis, A. Lazarus

Department of Mechanical Engineering, Massachusetts Institute of Technology, Cambridge MA 02139, USA,

E-mail: preis@mit.edu

P. M. Reis

Department of Civil and Environmental Engineering, Massachusetts Institute of Technology, Cambridge MA 02139, USA

A. Lazarus: Current address: Sorbonne Universités, UPMC Univ Paris 06, CNRS, UMR 7190 Institut Jean Le Rond D'Alembert, F-75005 Paris, France.

[**] We thank Denis Terwagne for help with the measurements of the Young's modulus of the elastomers. A.L. thanks the Battelle-MIT program for a postdoctoral fellowship. P.M.R. acknowledges support from the National Science Foundation CMMI-1351449 (CAREER). and $b$, respectively. Depressurizing our elastomeric structures allows for reversible control of the resulting motion, on demand. Below a critical pressure, there is cooperative local buckling of the thin ligaments that neighbor the voids, which themselves can be selectively plugged with inclusions. A void with (or without) an inclusion is regarded as a mechanical pixel, or mexel, that can be off (or on) to preclude (or enable) local buckling. The underlying auxetic behavior causes a a)

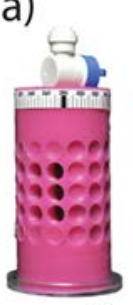

$\eta=0$

b)

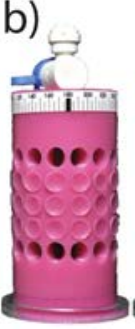

$\eta=0$

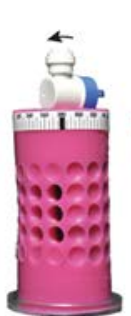

0.17

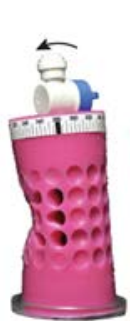

0.32
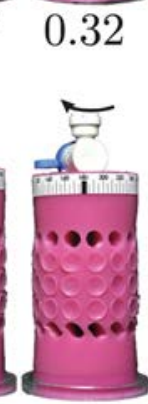

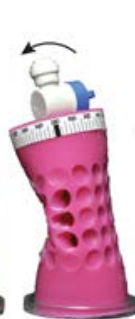

0.49
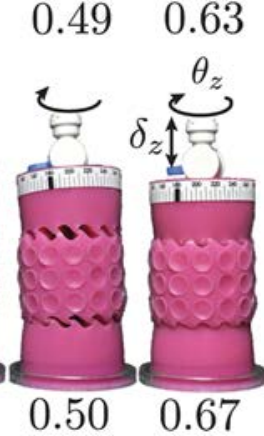

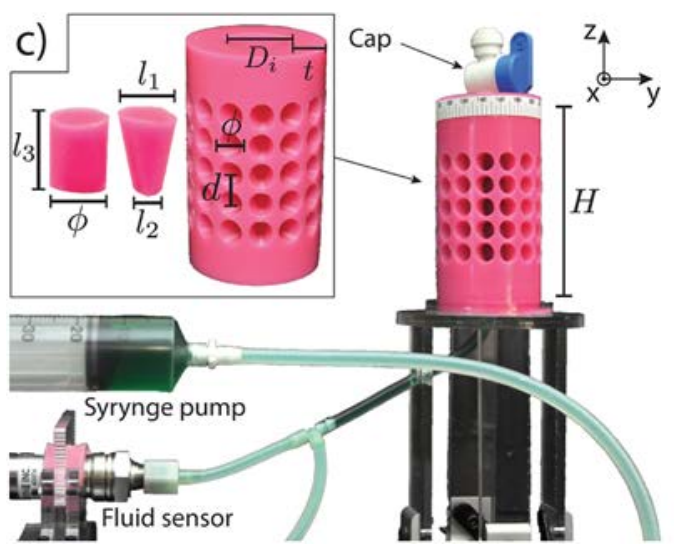

Fig. 1. Soft actuation of structured cylinders through auxetic behavior. (a) Flexural and (b) twisting actuation of representative samples, at increasing values of the dimensionless pumped volume, $\eta$. (c) Photograph of the experimental apparatus. Inset: Geometric parameters of the sample and the elastomeric conical plugs (with elliptical cross-section) used for the off (filled void) mechanical pixels, or mexels. 
localized contraction, which can be modified by the layout of mexels. In turn, this leads to global buckling of the sample that can be modulated to target specific modes of deformation. We make use of precision model experiments to explore the mechanical response of our structures and systematically study the layout of mexels to map out the regions of stability of flexural and twisting motion.

In Figure 1c, we present a photograph of our apparatus. A cylindrical sample was clamped to a supporting base and connected to a hydraulic system. The samples (Figure 1c, inset) were thick elastomeric cylindrical shells (internal diameter $D_{i}=25 \mathrm{~mm}$ and wall thickness $t=13.8 \mathrm{~mm}$ ) cast out of a silicone-based elastomer (vinylpolysiloxane [VPS]) and patterned with a square array of voids, around their circumference. See Experimental Section for fabrication details. The structure was made watertight by a thin membrane (thickness $h=0.4 \pm 0.1 \mathrm{~mm}$ ) that covered the inner surface of the voids. The sample was then fully filled with water (an incompressible fluid). Loading was performed hydraulically, under controlled volume conditions, using a syringe pump at a rate of $27 \mathrm{ml} \cdot \mathrm{min}^{-1}$. Starting from the undeformed configuration, each experiment consisted of a loading phase, where a volume $V$ of water was pumped out of the system up to a maximum value, followed by an unloading phase, where that same amount of fluid was re-injected. The primary control parameter is taken to be the dimensionless pumped volume, $\eta=V / V_{0}$, normalized by the initial internal volume of the cylinder $\left(V_{0}=41 \mathrm{ml}\right.$ for the samples with a height $H=10 \mathrm{~cm}$ ). We characterized the mechanical response of our structures by measuring the in-out differential pressure, $\Delta p$, and monitored the resulting deformation by two orthogonal digital cameras. Figure $1 \mathrm{a}$ and $\mathrm{b}$ show representative examples of flexural and twisting deformation, respectively, as $\eta$ is increased.

We start by investigating the response of an axisymmetric sample containing a regular $5 \times 14$ square array of voids around the full circumference of the cylinder. In Figure 2a, we present a series of photographs of representative configurations during the loading phase, at increasing values of the dimensionless pumped volume, from $\eta=0$ to 0.73 . Decreasing the internal pressure of the sample (i.e., increasing the in-out pressure differential, $\Delta p$ ) first causes the thin membranes that cover the voids to snap-buckle and invert their curvature inwards. This is followed by a period of axisymmetric radial compression of the walls (Figure $2 \mathrm{a}, \eta=0.24$ ). Eventually, the ligaments between the voids cooperatively buckle on the surface of the cylinder and the original regular square pattern of voids morphs into an arrangement of alternating and mutually orthogonal ellipses, on two sides of the cylinder. During this stage, there is loss of axisymmetry due to global buckling; the sample becomes increasingly flattened and the elliptical voids eventually close (Figure $2 \mathrm{a}, \eta=0.73$ ), analogously to that previously observed on flat $2 \mathrm{D}$ periodic elastomeric slabs. ${ }^{[6]}$

In Figure $2 b$, we plot the in-out differential pressure, $\Delta p$, versus $\eta$, for the originally axisymmetric samples described above, with three values of the elastomer Young's modulus,

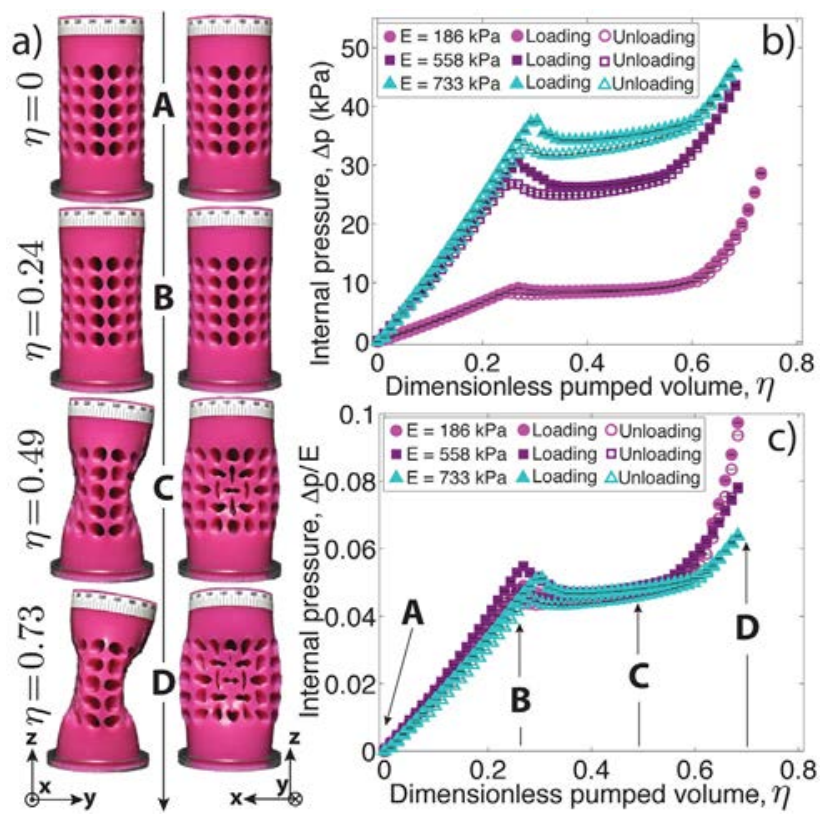

Fig. 2. Mechanical response of axisymmetric samples. (a) Orthogonal side views (onto the $y-z$ and $x-z$ planes of Figure $1 c$ ) for a sample with $E=186 \mathrm{kPa}$, for four consecutive stages of the loading path at $\eta=V / V_{0}=(0,0.24,0.49,0.73)$. (b) In-out pressure differential, $\Delta p$, versus dimensionless pumped volume, $\eta$, for three samples with different values of the Young's modulus, $E=(186,558,733) \mathrm{kPa}$. (c) $\Delta p$ nondimensionalized by $E$, for the same data shown in (b).

$E=(186,558,733) \mathrm{kPa}$. Both the loading and unloading paths are shown (open and closed symbols, respectively). For small values of $\eta$, there is a linear relation between $\Delta p$ and $\eta$ with a positive slope, i.e., a positive structural stiffness. At $\eta_{\mathrm{c}}=(0.27$, $0.27,029)$, for the above three values of $E$, respectively, there is a peak in $\Delta p$, after which the sample exhibits negative structural stiffness but eventually the internal pressure ramps up again. This response is reversible and the structure recovers fully to its original shape during unloading (our samples are made out of an elastic material), albeit with a hysteresis near $\eta_{\mathrm{c}}$ that may be attributed to the elastic snapthrough of the initially curved walls of the cylinder. In Figure 2c, we normalize $\Delta p$ by $E$ of the elastomer used, and the data collapse onto a master curve that further emphasizes the scale invariance of the underlying elastic buckling. The particular direction along which the sample buckles and then flattens is presumably triggered and chosen randomly by material imperfections frozen during the fabrication process, making the process challenging to control.

We now devise a strategy for controllable motion that overcomes this imperfection sensitivity by introducing pronounced geometric imperfections to target specific global buckling modes. To do so, a number of voids in the pattern layout were filled with inclusions during casting (using the same elastomeric material as the rest of the sample; see Figure 1c, inset), to preclude local buckling. We regard each void, with/without an inclusion, as a mechanical pixel, or mexel, which can be off/on, to prevent/enable local buckling, respectively. When two adjacent mexels are set to on, the 

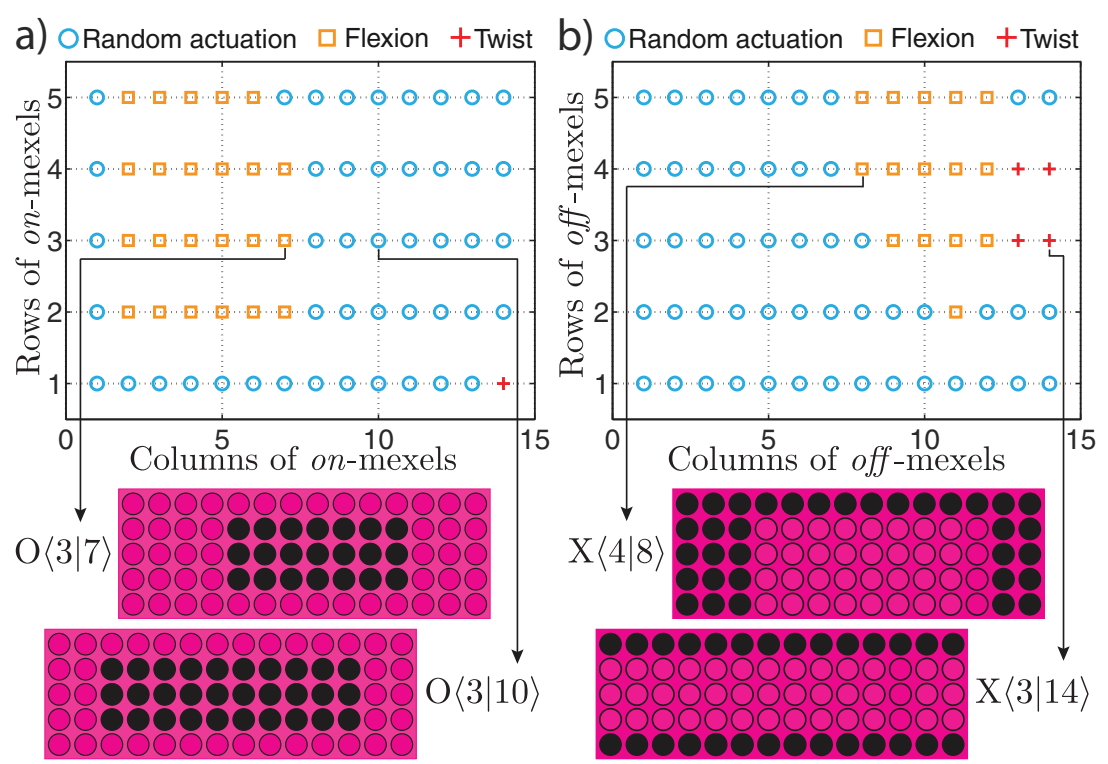

Fig. 3. Phase diagrams for different layouts of mechanical pixels. (a) Layouts with $m \times n$ arrays of on-mexels (voids), denoted by $O\langle m \mid n\rangle$. Bottom: schematic examples for $O\langle 3 \mid 7\rangle$ (flexion mode) and $\mathrm{O}\langle 3 \mid 10\rangle$ (random actuation). (b) Layouts with $m \times n$ arrays of off-mexels (filled), denoted by X $\langle m \mid n\rangle$. Bottom: schematic examples for X $\langle 4 \mid 8\rangle$ (flexion mode) and $X\langle 3 \mid 14\rangle$ (twist mode). Samples made out of VPS with Young's modulus $E=186 \mathrm{kPa}$. In the schematic layouts, a black circle represents an on-mexel and a filled (pink) circle represents an off-mexel.

shared ligament is free to buckle. We study two types of patterns with a $m \times n$ rectangular array of either (i) a patch of on-mexels within a region of off-mexels or (ii) vice versa. The corresponding configurations are denoted by $O\langle m \mid n\rangle$ and $X\langle m \mid n\rangle$, respectively. For simplicity, we focus on samples with $E=186 \mathrm{kPa}$.

The $O\langle 3 \mid 7\rangle$ configuration (schematic in Figure 3a) has three rows and seven columns of on-mexels, with all other units set to off (filled voids). Under loading, the ligaments of the onmexels buckle locally, thereby contracting one side of the cylinder and the sample flexes with a rotation around the $x$ axis (while remaining static around the $y$-axis). In Figure 1a, we presented a sequence of representative snapshots of this hinge-like motion at different values of the control parameter, $\eta$. The direction of flexion is set directly by the location of the patch. The $X\langle 4 \mid 8\rangle$ configuration (schematic in Figure $3 b$ ) exhibits a similar mode deformation, which we classify as the "flexion" regime.

By contrast, the $O\langle 3 \mid 7\rangle$ configuration (schematic in Figure 3a) is similar to the case presented earlier in Figure 2; the pattern transformation is sensitive to imperfections and the direction of deformation is chosen randomly by material defects. Given the difficulty in controlling the resulting global motion, we classify this as the "random actuation" regime.

When isolated rows of on-mexels are introduced around the full circumference of the sample (e.g., the $X\langle 3 \mid 14\rangle$ configuration shown in Figure $3 b$ ) twisting ensues, as shown in Figure $1 \mathrm{~b}$ for increasing values of $\eta$. Above a critical load, there is a synchronized local buckling in a common direction (set by material defects) of the initially parallel free ligaments and shear bands form. These result in a screw-like motion of the cylinder with a rotation around the $z$-axis, coupled with axial contraction. We classify this as the "twist" regime.

We now systematically explore the response for various arrangements of mexels and classify the resulting motion according to the three regimes introduced above: (i) random actuation, (ii) flexion, and (iii) twist. The corresponding phase diagrams are presented in Figure $3 a$ for $O\langle m \mid n\rangle$ and in Figure $3 \mathrm{~b}$ for $X\langle m \mid n\rangle$, where a particular data point represents the number of rows and columns of the on and off mexels, respectively. In both cases, there are extended regions of the design space for which we find a flexion (square symbols). Moreover, out of the configurations explored, the following exhibit twist: $O\langle 1 \mid 14\rangle, X\langle 3 \mid 13\rangle, X\langle 3 \mid 14\rangle, X\langle 4 \mid 13\rangle$, and $X\langle 4 \mid 14\rangle$. The remaining configurations result in random actuation (circle symbols), for cases that are either under-constrained (onmexels cover more than half of the circumference of the cylinder) or over-constrained (patches of on-mexels smaller than $2 \times 2$ ). The under-constrained cases are sensitive to material imperfections and exhibit global buckling with a motion that is difficult to control.

We proceed by focusing on the flexion and twist regimes and characterize the mechanical response of representative $O\langle m \mid n\rangle$ and $X\langle m \mid n\rangle$ arrangements by measuring the internal pressure, $\Delta p$, and the amplitude of motion (flexure or twist), as a function of the dimensionless pumped volume ratio, $\eta$.

In Figure $4 \mathrm{a}$, we plot $\Delta p$ versus $\eta$ for four different configurations $O\langle 3 \mid i\rangle$, with $i=(4,5,6,7)$, in the flexion regime. Similarly to the axisymmetric case above (Figure $2 b$ ), the behavior of the $\Delta p(\eta)$ curves is reversible (despite some hysteresis) with two distinct regimes, separated by the onset of pattern transformation at $\eta_{\mathrm{c}}=0.22$, which itself is nearly independent across the four samples. The initial linear regime 

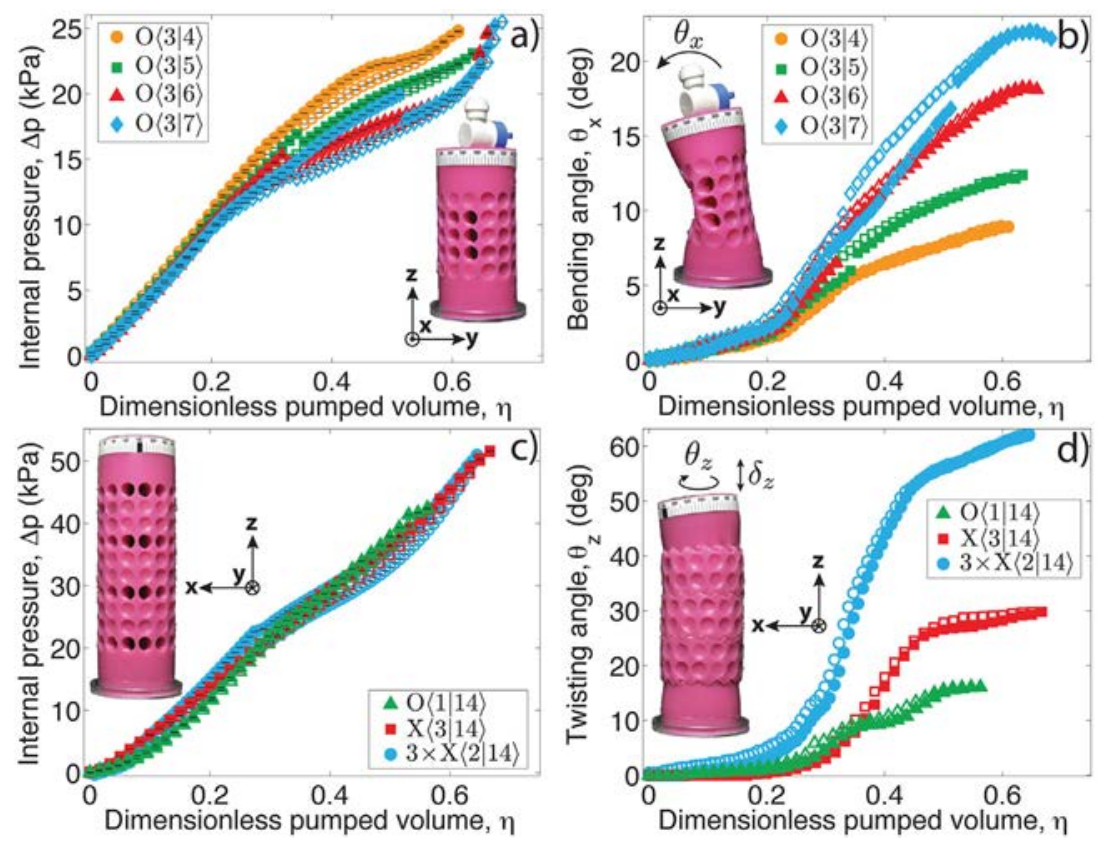

Fig. 4. Mechanical response and kinematics of actuation. (a) In-out pressure differential, $\Delta p$, versus dimensionless pumped volume, $\eta$ and (b) angle of flexion, $\theta_{x}$, versus $\eta$ for the following configurations: $O\langle 3 \mid 4\rangle, O\langle 3 \mid 5\rangle, O\langle 3 \mid 6\rangle$, and $O\langle 3 \mid 7\rangle$. The maximum values of the flexure angle attained are $\theta_{x}=\left(9^{\circ}, 12^{\circ}, 18^{\circ}, 22^{\circ}\right)$, respectively. $(c)$ Internal pressure, $\Delta p$ versus $\eta$ and (d) twisting angle, $\theta_{z}$, versus $\eta$ for the $O\langle 1 \mid 14\rangle, X\langle 3 \mid 14\rangle$, and $3 \times X\langle 2 \mid 14\rangle$ configurations. The maximum values of the twisting angle attained are $\theta_{z}=\left(16^{\circ}, 30^{\circ}\right.$, $\left.62^{\circ}\right)$, respectively. Loading path (filled symbols), unloading path (open symbols). Samples made out of VPS with Young's modulus $E=186 \mathrm{kPa}$.

with approximately the same slope across the four samples is followed by a post-buckling regime that depends on the arrangement of on-mexels. We note that $\Delta p$ increases monotonically after $\eta_{c}$, in contrast with the axisymmetric case that exhibited a region of negative slope past the instability. The positive structural stiffness throughout the deformation process for these $\mathrm{O}\langle 3 \mid i\rangle$ configurations is significant of a load bearing capacity, which is increased for samples with fewer on-mexels, albeit at the cost of reduced flexure motion, as we discuss next.

The flexure motion is quantified by the angle, $\theta_{x}$, around the $x$-axis, that the normal of the top surface of the sample makes with the vertical. In Figure $4 \mathrm{~b}$, we plot $\theta_{x}$ versus $\eta$ for the $O\langle 3 \mid i\rangle$ configurations discussed above. The $\theta_{x}(\eta)$ curves also exhibit two regimes. Prior to $\eta_{c}, \theta_{x}$ evolves linearly with $\eta$, after which it increases steeply and nonlinearly. By controlling the internal volume of the sample, $\theta_{x}$ can be changed smoothly up to the maximum value, which was $\theta_{x}=\left(9^{\circ}, 12^{\circ}, 18^{\circ}, 2^{\circ}\right)$ for $O\langle 3 \mid i\rangle$ with $i=(4,5,6,7)$, respectively.

We turn to characterizing three representative samples in the twist regime: $O\langle 1 \mid 14\rangle, X\langle 3 \mid 14\rangle$ and $3 \times X\langle 2 \mid 14\rangle$. The third is fabricated by taking $X\langle 2 \mid 14\rangle$ as a building block and vertically stacking three such configurations; the resulting $3 \times X\langle 2 \mid 14\rangle$ sample comprises four full circumferential rows of on-mexels, each separated by two consecutive rows of offmexels (see photographs in Figure $4 \mathrm{c}$ and $d$, insets). In order to fully uncouple the local buckling of the ligaments and the global buckling mode, we have rigidified the wall in the region of the off-mexels by adding an internal acrylic brace (note that, instead, we could have increased the wall thickness). For all three samples, $\Delta p$ increases monotonically with $\eta$ (Figure $4 \mathrm{c}$ ) with a pre- and post-buckling regime separated at $\eta_{\mathrm{c}} \approx 0.28$. The rotation resulting from the screwlike motion is quantified by the twist angle, $\theta_{z}$, around the $z$ axis, which we plot in Figure $4 \mathrm{~d}$ as a function of $\eta$. The angular motion is linear up to $\eta_{c}$, after which it increases steeply and eventually levels off for higher values of $\eta$ (once the on-mexels are fully closed). It is interesting to note that the maximum value of $\theta_{z}$ attained is directly proportional to the number of isolated rows of on-mexels, each of which contributes with a maximum of $15^{\circ}$ to the global twist motion.

In conclusion, we have identified two functional regimes in structured auxetic materials that lead to tunable and controllable flexure or twist, depending on their layout of mexels. Given that the deformation is primarily governed by the geometry of the design, coupled to the buckling of the thin ligaments of the pattern, the resulting modes of deformation should be readily scalable. Our structured cylindrical shells can be regarded as soft actuators with passive activators that are embedded directly onto their walls. Ultimately, it may be possible to control each of the mexels, or a cluster of them, independently and in an automated way, which would enable complex motion with a large number of degrees of freedom, analogously to an octopus-arm.

\section{Experimental Section}

\subsection{Fabrication of Samples}

The samples were cast out of VPS (Elite Double 8, 16 and 32 Zhermack), a silicone-based elastomer, using custom 
designed molds. The Young's modulus of the three elastomers used was measured, through standard uniaxial tensile testing using an Instron machine, to be $E=186,558$, and $733 \mathrm{kPa}$ ( $5 \%$ error), with a Poisson's ratio of $v \approx 0.5$. Molds were fabricated using a combination of laser-cutting and 3D-printing. Each mold consisted of two polymethylmethacrylate (PMMA) cylinders that were concentrically aligned and held together by two acrylic discs mounted at their extremities. The larger cylinder had an inner diameter $D=52.6 \mathrm{~mm}$, and the smaller cylinder had an outside diameter of $D_{i}=25 \mathrm{~mm}$. VPS was poured into the gap between the two, which resulted on samples of thickness, $t=\left(D-D_{i}\right) / 2$. The larger cylinder was itself periodically patterned with a square array of $5 \times 14$ laser-cut holes distributed equidistantly around its circumference. Each of these holes had a diameter of $\varphi=9.85 \mathrm{~mm}$, resulting in an inter-hole distance of $d=11.8 \mathrm{~mm}$, in both the longitudinal and tangential directions of the array. Two types of plugs were inserted into each of these holes to create the two possible mechanical pixels (mexels), on (void) or off (filled). For the on mexels, we 3D-printed conical pins with an elliptical cross-section of constant major diameter $\varphi=9.85$ $\mathrm{mm}$ and a minor diameter that varied along the height. This varying cross-section was necessary to account for the decrease of available surface area in the radial direction of the cylindrical sample. The dimensions of each pin was $\left(l_{1}, l_{2}\right.$, $\left.l_{3}\right)=(10.0,4.3,18.6) \mathrm{mm}$ (see inset of Figure $\left.1 \mathrm{c}\right)$. The height $l_{3}$ of the pin was such that, upon casting, it created a thin membrane of thickness $0.4 \pm 0.1 \mathrm{~mm}$ on the inner surface of the sample, which made it water tight. For the off (filled) pixels, the hole on the outer acrylic cylinder was capped using a $10 \mathrm{~mm}$ diameter steel pin, such that the distance from its surface to the inner cylinder was $t$, i.e., the void was fully filled with VPS.

\subsection{Mechanical Testing}

The sample was sealed and filled with water. The testing apparatus comprised a motorized syringe pump (NE-100, New Era Pump Systems) with a syringe $\left(60 \mathrm{~cm}^{3}\right.$, Irrigation Syringe) and the pressure was monitored by a sensor (PX409015V10V, Omegadyne), all connected using silicone tubing (51135k84, Mc Master-Carr). During each experimental run, the sample was hydraulically loaded/unloaded by withdrawing/injecting water under volume-controlled conditions, at a rate of $27 \mathrm{ml} \cdot \mathrm{min}^{-1}$. Imaging was performed using a SLR digital camera (D90, Nikon) aligned perpendicularly to the $x-z$ a or the $y-z$ planes (Figure 1c) and a series of photographs was acquired at every $0.5 \mathrm{ml}$ of volume of fluid pumped. The kinematic quantities such as flexural or twisting angles were measured using the image processing software ImageJ.
[1] R. Lakes, Science 1987, 235, 1038.

[2] R. Lakes, Adv. Mater. 1993, 5, 293.

[3] K. E. Evans, A. Alderson, Adv. Mater. 2000, 12, 617.

[4] J. N. Grima, K. E. Evans, J. Mater. Sci. Lett. 2000, 19, 1563.

[5] Y. Ishibashi, M. Iwata, J. Phys. Soc. Jpn. 2000, 69, 2702.

[6] T. Mullin, S. Deschanel, K. Bertoldi, M. Boyce, Phys. Rev. Lett. 2007, 99, 084301.

[7] K. Bertoldi, M. Boyce, S. Deschanel, S. Prange, T. Mullin, J. Mech. Phys. Solids 2008, 56, 2642.

[8] K. Bertoldi, P. M. Reis, S. Willshaw, T. Mullin, Adv. Mater. 2010, 22, 361.

[9] J. T. Overvelde, S. Shan, K. Bertoldi, Adv. Mater. 2012, 24, 2337.

[10] J. T. Overvelde, K. Bertoldi, J. Mech. Phys. Solids 2013, 64, 351.

[11] M. Taylor, L. Francesconi, M. Gerendás, A. Shanian, C. Carson, K. Bertoldi, Adv. Mater. 2013, 26, 2365.

[12] J. Shim, C. Perdigou, E. R. Chen, K. Bertoldi, P. M. Reis, Proc. Natl. Acad. Sci. USA 2012, 109, 5978.

[13] K.-J. Cho, J.-S. Koh, S. Kim, W.-S. Chu, Y. Hong, S.-H. Ahn, Int. J. Precis. Eng. Manuf. 2009, 10, 171.

[14] S. Kim, C. Laschi, B. Trimmer, Trends Biotechnol. 2013, 31, 287.

[15] R. Pfeifer, M. Lungarella, F. Iida, Science 2007, 318, 1088.

[16] D. Trivedi, C. D. Rahn, W. M. Kier, I. D. Walker, Appl. Bionics Biomech. 2008, 5, 99.

[17] R. Geryak, V. V. Tsukruk, Soft Matter 2014, 10, 1246.

[18] M. Calisti, M. Giorelli, G. Levy, B. Mazzolai, B. Hochner, C. Laschi, P. Dario, Bioinspir. Biomim. 2011, 6,036002 .

[19] I. A. Anderson, T. A. Gisby, T. G. McKay, B. M. O’Brien, E. P. Calius, J. Appl. Phys. 2012, 112, 041101.

[20] A. Sadeghi, L. Bewccai, B. Mazzolai, 2012 IEEE/RSJ International Conference on Intelligent Robots and Systems (IROS), 2012, pp. 4237-4242.

[21] T. Umedachi, R. Idei, K. Ito, A. Ishiguro, Artif. Life 2013, $19,67$.

[22] C. D. Onal, D. Rus, Bioinspir. Biomim. 1969, 8, 026003.

[23] S. Seok, C. D. Onal, R. Wood, D. Rus, S. Kim, 2010 IEEE International Conference on Robotics and Automation (ICRA), 2010, pp. 1228-1233.

[24] H.-T. Lin, G. G. Leisk, B. Trimmer, Bioinspir. Biomim. 2011, 6, 026007.

[25] R. F. Shepherd, F. Ilievski, W. Choi, S. A. Morin, A. A. Stokes, A. D. Mazzeo, X. Chen, M. Wang, G. M. Whitesides, Proc. Natl. Acad. Sci. USA 2011, 108, 20400.

[26] F. Ilievski, A. D. Mazzeo, R. F. Shepherd, X. Chen, G. M. Whitesides, Angew. Chem. 2011, 123, 1930.

[27] B. Mosadegh, P. Polygerinos, C. Keplinger, S. Wennstedt, R. F. Shepherd, U. Gupta, J. Shim, K. Bertoldi, C. J. 
Walsh, G. M. Whitesides, Adv. Funct. Mater. 2013, 24, 2163.

[28] S. A. Morin, S. W. Kwok, J. Lessing, J. Ting, R. F. Shepherd, A. A. Stokes, G. M. Whitesides, Adv. Funct. Mater. 2014, 24, 5541.

[29] E. Steltz, A. Mozeika, N. Rodenberg, E. Brown, H. Jaeger, IEEE/RSJ International Conference on Intelli- gent Robots and Systems (IROS), 2009, pp. 56725677.

[30] E. Brown, N. Rodenberg, J. Amend, A. Mozeika, E. Steltz, M. R. Zakin, H. Lipson, H. M. Jaeger, Proc. Natl. Acad. Sci. USA 2010, 107, 18809.

[31] C. Laschi, M. Cianchetti, B. Mazzolai, L. Margheri, M. Follador, P. Dario, Adv. Robot. 2012, 26, 709. 